\title{
Novel entropic elasticity of polymeric materials: why is slide-ring gel so soft?
}

\author{
Kohzo Ito
}

We have recently developed a slide-ring gel that is different from physical and chemical gels by crosslinking polyrotaxane, a necklace-like supramolecule. Slide-ring gel, consisting of movable crosslinks and free uncrosslinked cyclic molecules, shows peculiar mechanical properties, different from conventional chemical gels. For instance, slide-ring gel shows quite a low Young's modulus, which is not proportional to crosslinking density and is much lower than that of chemical gels with the same density. This low modulus arises from differences in the molecular mechanism of entropic elasticity; whereas conformational entropy is mainly responsible for elasticity in typical chemical gels and rubbers, the mechanical properties of slide-ring gel are inherently governed by the arrangement entropy of free cyclic molecules in polyrotaxane, as well as the conformational entropy of the axis polymer. This means that the softness of slide-ring gel is due to novel entropic elasticity, which is also expected to provide a sliding state and a sliding transition.

Polymer Journal (2012) 44, 38-41; doi:10.1038/pj.2011.85; published online 14 September 2011

Keywords: crosslink; entanglement effect; entropic elasticity; polyrotaxane; pulley effect; slide-ring gel; sliding state

\section{INTRODUCTION}

Since the discovery of crosslinking in natural rubber with sulfur in 1839 by Goodyear, crosslinking of polymeric materials has been one of the most important topics in polymer science and technology. ${ }^{1,2}$ Uncrosslinked natural rubbers inherently resemble liquids with regard to their flow behavior, although they show viscoelastic properties due to the entanglement of polymer chains. Once crosslinked, however, they behave like solids, in that they maintain their shape against deformation above the glass temperature. Thus far, the unique mechanical behavior of crosslinked polymeric materials has been investigated intensively. ${ }^{1,2}$ The elasticity of crosslinked rubbers and elastomers arises mainly from the conformational entropy of polymer chains between crosslinking junctions, with Young's modulus increasing proportionally with crosslinking density. Entropic elasticity also gives a stable rubber state characteristic of crosslinked polymeric materials.

Polymer entanglement is also one of the most important features of polymers. It behaves like crosslinking over short time scales, but entangled chains are eventually released by reptation or one-dimensional diffusion along a polymer chain. Accordingly, reptation of uncrosslinked chains relaxes the rubber elasticity of the entangled polymer system over a relaxation time. The entanglement effect is described effectively by the tube or reptation model, developed by de Gennes ${ }^{3}$ and Edwards and $\mathrm{Doi}^{4}$ in the 1970s, in which each chain is assumed to move along a tube, due to constraints imposed by surrounding chains. The tube model has enabled us to understand the relationship between molecular structure and linear rheology in an entangled system. ${ }^{5}$ Alternatively, Ball et $a .^{6}$ proposed a slip-link model, in which the chain can slip at a trapped entanglement. The slip-link model is widely used in computer simulations of viscoelastic behavior in uncrosslinked and crosslinked polymeric materials.

We have recently developed a new crosslinking structure using polyrotaxane (PR), ${ }^{7}$ a necklace-like supramolecule, in which cyclic molecules are threaded onto an axis polymer chain. ${ }^{8}$ We prepared PR containing a small amount of $\alpha$-cyclodextrin $(\alpha-\mathrm{CD})$ on polyethylene glycol and subsequently crosslinked $\alpha$-CDs onto different PRs. As a result, the crosslinking junctions of the figure-eight shapes were not fixed and could move freely in the polymer network. The polymer network also contained free cyclic molecules, which remained uncrosslinked. We refer to this new crosslinked polymer network as a slidering gel (Figure 1). Such a polymeric material, with freely movable crosslinks that resemble the slip-link model, was considered theoretically as a sliding gel by de Gennes in $1999 .{ }^{9}$ Slide-ring gel, with its movable crosslinks and free uncrosslinked cyclic molecules, shows peculiar mechanical properties, different from conventional chemical gels. For instance, slide-ring gel shows quite a low Young's modulus compared with that of chemical gels with the same crosslinking density. The concept of freely movable crosslinks is not limited to slide-ring gels containing solvents, but has already been applied to slide-ring materials without solvents. In fact, a slide-ring elastomer showing scratch-proof properties has recently been used as a coating material on a mobile phone.

In this study, we consider why slide-ring gel is so soft compared with chemical gels and what determines the Young's modulus of 


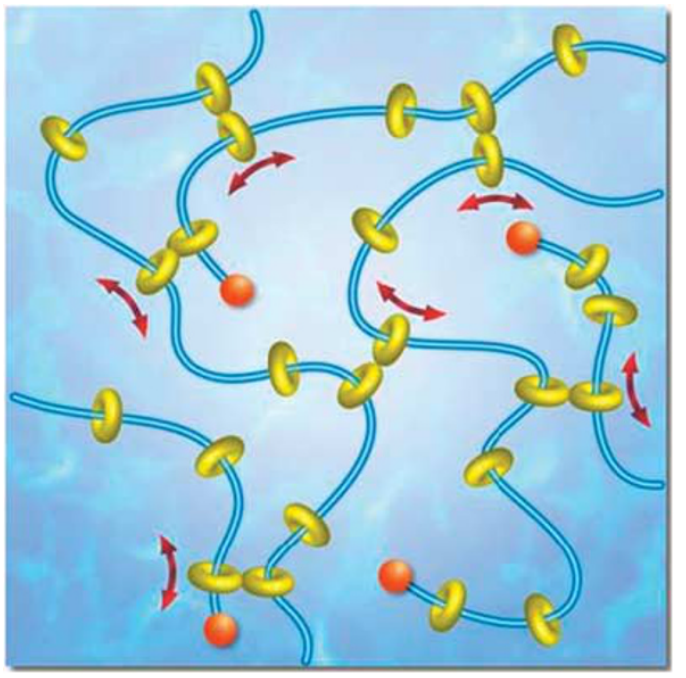

Figure 1 Schematic diagram of slide-ring gel with freely movable figure-eight crosslinks functioning like pulleys, where free cyclic molecules slide actively along axis polymer chains between crosslinks.

slide-ring gel. The movable crosslinking junctions in slide-ring gels gives mechanical behavior, quite different from the entanglement of polymer chains or the fixed junctions in chemical gels and rubbers. The polymer chain in slide-ring gel can pass through the crosslink but does not dissociate from it completely, due to topological restrictions. In addition, free cyclic molecules that remain uncrosslinked in PR have arrangement entropy on axis polymer chains, whereas conventional polymeric materials have no such entropy. The elasticity of chemical gel or rubber arises mainly from the conformational entropy of the polymer chains. Thus, the arrangement entropy of free cyclic molecules may affect the elasticity or mechanical properties of slide-ring gel.

\section{MECHANICAL PROPERTIES OF SLIDE-RING GEL}

It is well known that the Young's modulus $E$ of crosslinked polymeric materials such as chemical gel or rubber is given by the affine model, $E=v k T$, where $v$ is the number density of elastically effective network strands and $k T$ is the thermal energy. ${ }^{1,2}$ The phantom model gives an estimate one-third or one-half of it. ${ }^{1,2}$ However, slide-ring gel does not obey this rule. Figure 2 shows the dependence of Young's modulus in slide-ring and chemical (pullulan) gels on crosslinker concentration, where gelation with $1,1^{\prime}$-carbonyldiimidazole was performed in a $20 \%$ dimethyl sulfoxide solution of pullulan (MW $=30-50 \mathrm{kDa})$ at $80^{\circ} \mathrm{C}$ for $36 \mathrm{~h}$ or in a $10 \%$ dimethyl sulfoxide solution of polyrotaxane $(\mathrm{MW}=105 \mathrm{kDa})$ of polyethylene glycol $(\mathrm{MW}=35 \mathrm{kDa})$ and $\alpha$-CD with an inclusion ratio of $27 \%$ at $50{ }^{\circ} \mathrm{C}$ for $96 \mathrm{~h} .{ }^{10}$ This means that the volume fraction of the pullulan gel was as much as that of the slide-ring gel. The Young's moduli were determined with small-amplitude oscillatory compression measurements, which showed no frequency dispersion over a range of $0.01-10 \mathrm{~Hz}$. Although the chemical gel modulus increased proportionally with crosslinker concentration along the slope expected from the standard model, the modulus of the slide-ring gel deviated from it considerably and was much lower than that of the chemical gel. Incidentally, the actual crosslinking density may be less than that estimated from the crosslinker concentration because the total amount of crosslinker should not contribute to the crosslinking reaction between different polymers and produce crosslinking junctions. This probably explains why the

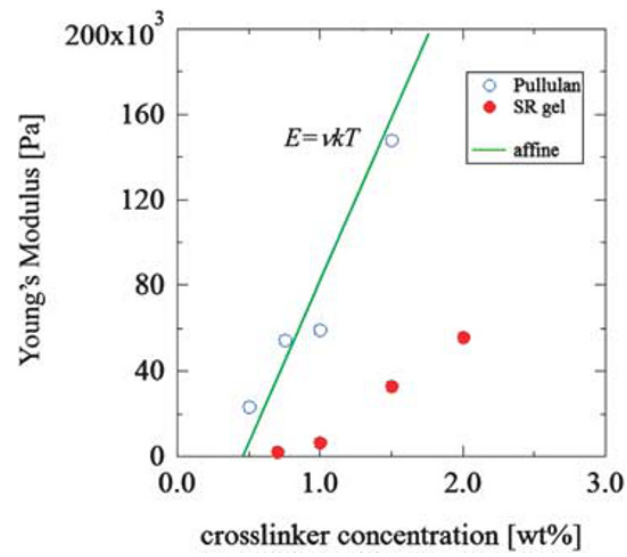

Figure 2 Dependence of Young's modulus of slide-ring gels on concentration of crosslinker chemical (1,1'-carbonyldiimidazole). Young's modulus increases linearly with the crosslinker concentration in chemical gel, whereas slide-ring gel shows a much lower modulus that is not proportional to concentration.

dependence of Young's modulus on crosslinker concentration in the pullulan gel does not pass through the origin.

Figure 3a shows the dependence of the stress-strain curve on the solvent species in slide-ring gels prepared in the same way as mentioned above, with a crosslinker concentration of $0.75 \% .{ }^{10}$ Because $\alpha$-CDs in PR are sparsely distributed in dimethyl sulfoxide (DMSO), ${ }^{11}$ stress increases linearly with extension ratio owing to the pulley effect, which results in a J-shaped curve over a wider strain range. ${ }^{12}$ By contrast, $\alpha$-CDs in PR form aggregates like nodules in a poorer solvent such as water by hydrogen bonding between hydroxyl groups on $\alpha$-CDs, as shown in Figure $3 b,{ }^{7}$ thereby suppressing the pulley effect considerably. There, the stress-strain curve is similar to that of typical chemical gels or a crosslinked rubber with fixed junctions, that is, an S-shaped one. ${ }^{1,2}$ Note that the Young's modulus increases roughly 30 fold where the changing solvent restrains the pulley effect. This indicates that the low Young's modulus of slide-ring gel results from the pulley effect. Incidentally, the volume of the gels in Figure 3 was almost independent of the solvent in the equilibrium swelling state, where the mechanical measurements were performed.

\section{WHAT DETERMINES THE YOUNG'S MODULUS OF SLIDE-RING GEL?}

It is well known that typical chemical gels and rubbers have finite equilibrium moduli (over long time scales), arising from the entropic elasticity of polymer conformation between fixed crosslinks. By contrast, entangled polymer chains, like liquids, have no finite equilibrium modulus because the release or reptation of entangled polymers eventually relaxes the chain deformation responsible for entropic elasticity. Slide-ring gel shows a sliding motion of polymer chains at the crosslinking junctions, similar to the entangled polymer system. Why does slide-ring gel show finite equilibrium at a low Young's modulus? Certainly, this is because the polymer chain can slide but is not released by the topological restriction; the stopper of PR at both ends hinders perfect dissociation of the axis polymer chain from the crosslinks. Then, what determines the low Young's modulus of slide-ring gel?

Recent experimental results show that a number of $\alpha$-CDs remain uncrosslinked in slide-ring gel. ${ }^{13}$ These $\alpha$-CDs show a sliding motion along the axis polymer in PR, yielding arrangement entropy. It should be stressed that these free cyclic molecules have a dominant role in the Young's modulus of slide-ring gel. Figure 4 shows the molecular mechanism of elasticity in slide-ring gel schematically. When slide- 


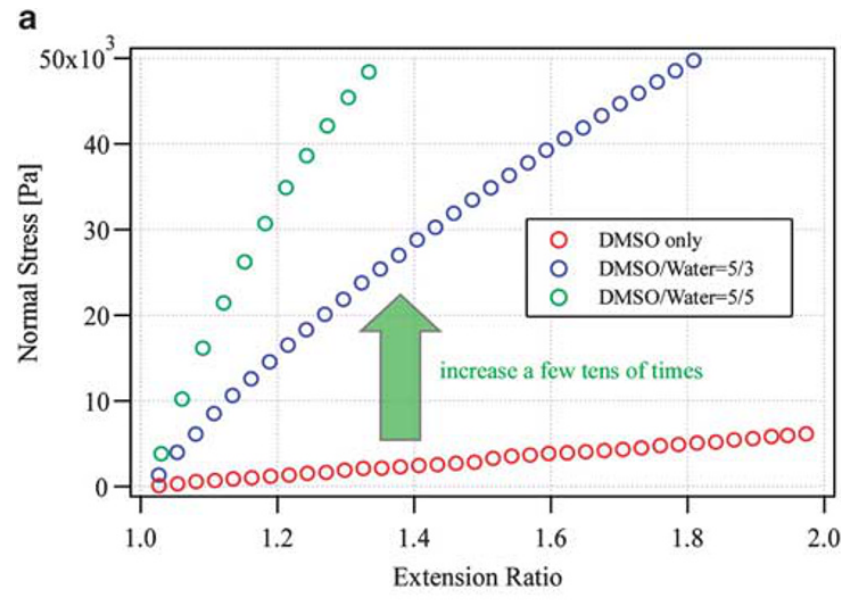

b

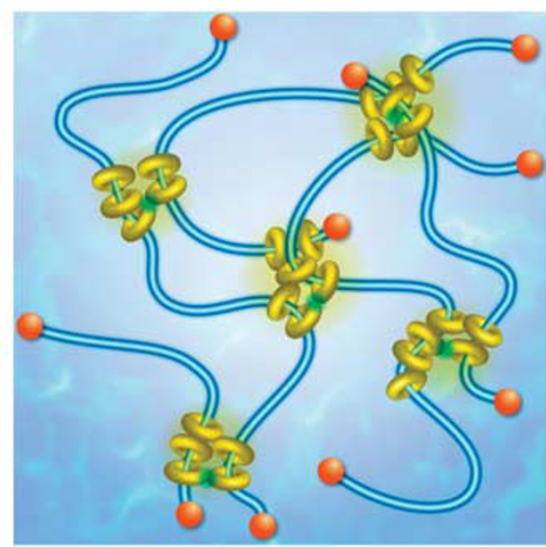

Figure 3 (a) Stress-strain curve of slide-ring gel with different solvents. Young's modulus increases roughly 30 -fold by adding water. The pulley effect is probably suppressed considerably in the mixture of dimethyl sulfoxide and water, owing to aggregation of cyclodextrins (CDs). (b) Illustration of CDs in slide-ring gel forming aggregations like nodules in a poor solvent.

ring gel is stretched along the horizontal axis, polymer chains are deformed to an anisotropic conformation in the short time scale. As time passes, the axis polymer relaxes the polymer deformation by sliding at the crosslinks, which is called the pulley effect. ${ }^{8}$ Small-angle $\mathrm{X}$-ray scattering on uniaxial deformation indicates relaxation of conformational anisotropy in slide-ring gel. ${ }^{14}$ However, free cyclic molecules cannot pass through crosslinks consisting of other rings of the same size, whereas axis polymer chains can easily do so. As a result, axis polymer sliding provides a heterogeneous density distribution of free cyclic molecules as the axis polymer chains relax conformation anisotropy. This heterogeneous distribution reduces the arrangement entropy of free molecules drastically, which leads to entropic elasticity completely different from that ascribed to the polymer conformation. The finite equilibrium low modulus of slide-ring gel should come from this novel kind of entropic elasticity due to the arrangement of free cyclic molecules on crosslinked PR.

\section{SLIDING STATE AND SLIDING TRANSITION}

Recent experimental results from neutron spin echo indicate that the time scale of $\mathrm{CD}$ sliding is much slower than the micro-Brownian motion of the polyethylene glycol segment. ${ }^{15}$ This means that polymer deformation should occur much faster than either the sliding relaxation of the axis polymer chain by the pulley effect or the sliding
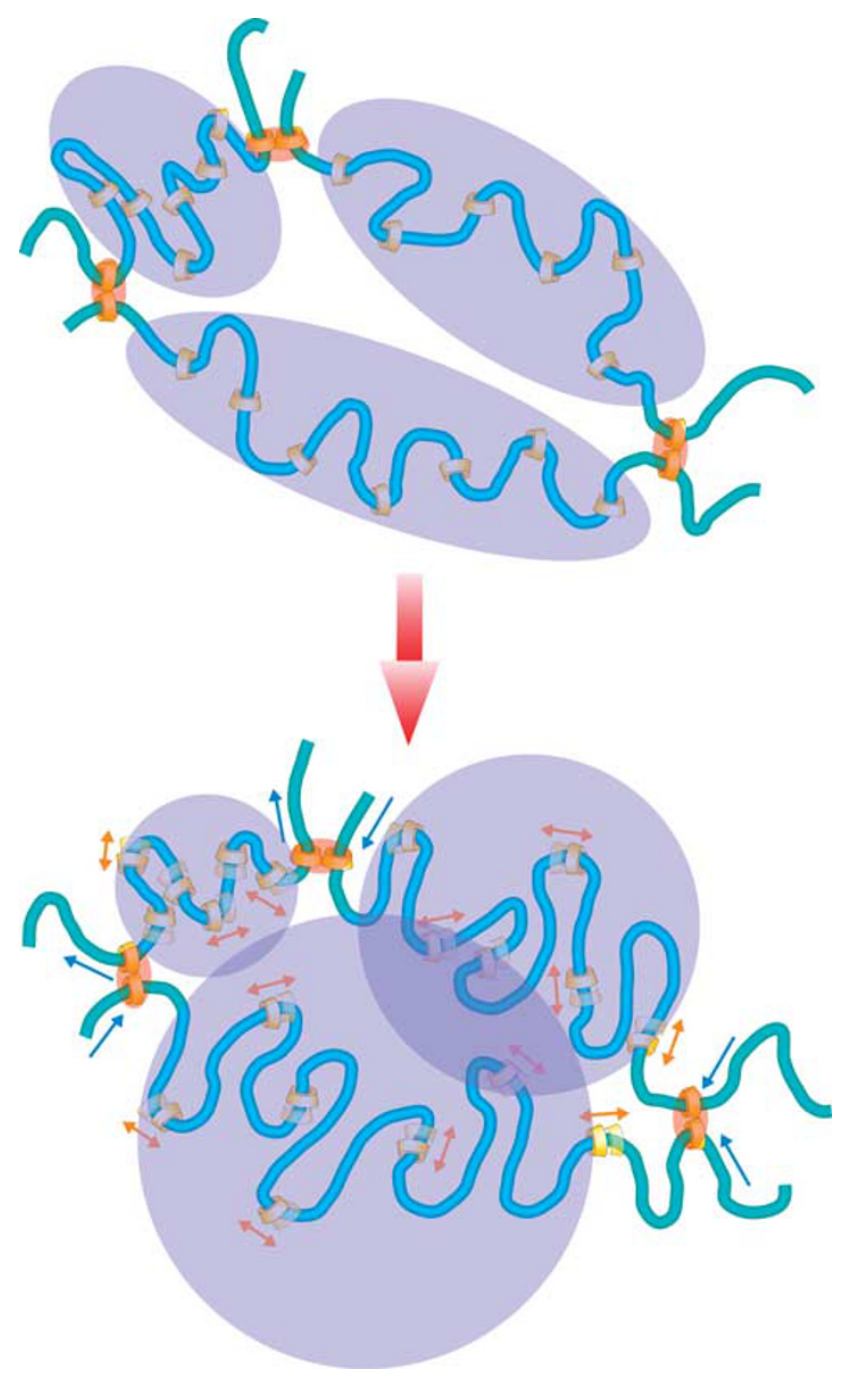

Figure 4 Relaxation of the rubber state to the sliding one by the sliding of axis polymer chains at crosslinking junctions. Polymer sliding relaxes the conformation anisotropy of axis polymer chains. Free cyclic molecules then form a heterogeneous density distribution because they cannot pass through crosslinks consisting of other rings of the same size.

motion of free cyclic molecules. From these results, we suggest dynamics for slide-ring gel, as shown schematically in Figure 5. Typical crosslinked amorphous polymers show glass and rubber states, divided by the glass transition. Slide-ring gel ought to have another state, which we have termed the 'sliding state, where axis polymer chains and cyclic molecules are sliding actively. The sliding state appears in regions of higher temperature or lower frequency than the rubber state because the sliding motion of free $\alpha$-CDs is slower than the microBrownian motion of the polymer segment. These two states are separated by the sliding transition, similar to the glass transition between glass and rubber states. The sliding transition reflects the time scale when axis polymer chains and cyclic molecules start sliding.

If arrangement entropy dominates sliding elasticity, modulus should increase linearly according to how many cyclic molecules are included in PR. This means that the sliding elasticity is similar to a one-dimensional air spring, in which air molecules are confined in a one-dimensional tube. In slide-ring gel, the sliding motion of free cyclic molecules threaded onto a chain is restricted within movable crosslinking junctions. If the sliding elasticity is larger than the rubber 


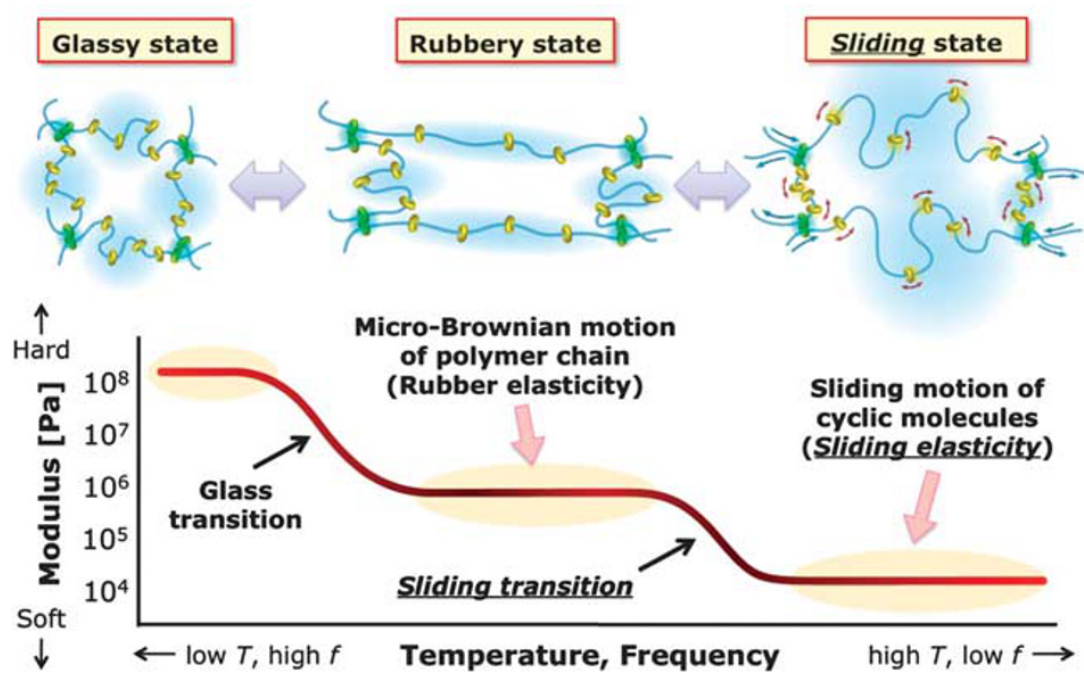

Figure 5 Mechanical dynamics of slide-ring materials. Typical crosslinked amorphous polymers show a glass transition between glass and rubber states, whereas slide-ring material shows a sliding transition and sliding state. In the sliding state, axis polymer chains and cyclic molecules are sliding actively. The sliding state should appear in regions of higher temperature or lower frequency than the rubber state.

one, the sliding transition and sliding state should disappear because the polymer chain can deform easily rather than forming a heterogeneous density distribution of free cyclic molecules by polymer sliding. Incidentally, sliding elasticity is not characteristic of uncrosslinked PR, but it is characteristic of crosslinked PR because the sliding motions of polymer and free cyclic molecules do not give a heterogeneous density distribution of free molecules upon deformation of uncrosslinked slide-ring materials. Uncrosslinked PRs probably behave as typical entangled or unentangled polymer chains as far as the mechanical properties are concerned.

\section{CONCLUDING REMARKS}

Since its discovery by Goodyear in 1839, crosslinking has been considered to be fixed at the polymer chains. Rubber elasticity was later well explained by the fixed junction model, by assuming affine deformation of the polymer chains. Slide-ring gel, however, has movable crosslinking and free cyclic molecules. The pulley effect, caused by sliding of the axis polymer at crosslinking junctions, relaxes stress due to polymer deformation, similar to reptation in the entangled polymer system. Instead of relaxation of the polymer deformation by sliding, free cyclic molecules form a heterogeneous density distribution, which results in substantial entropy loss. In other words, entropy loss from the axis polymer deformation transfers to that of the heterogeneous distribution of free cyclic molecules. As a result, sliding elasticity appears in the sliding state, where axis polymer chains and cyclic molecules are sliding actively. In addition, slide-ring gel should show a sliding transition between the rubber and sliding states. These aspects indicate that the unique mechanical characteristics of slide-ring gel arise from its dynamics. Slide-ring materials seem to give rise to a new field between crosslinking and entanglement in future polymer science and technology.

\section{ACKNOWLEDGEMENTS}

I thank my colleagues, Dr Kazuaki Kato, Mr Koichi Mayumi, Dr Yasuhiro Sakai and Professor Kenji Urayama, for helpful discussion. This work was partially supported by a Grant-in-Aid for Scientific Research ((S), 2008-2012, No. 20221005) of Japan.

1 Treloar, L. R. G. The Physics of Rubber Elasticity, 3rd edn. (Oxford University Press, Oxford, 1975).

2 Mark, J. E. \& Erman, B. Rubber Elasticity: A Molecular Primer 2nd edn. (Cambridge University Press, Cambridge, 2007).

3 de Gennes, P. G. Scaling Concept in Polymer Physics (Cornell University Press, Ithaca, 1979).

4 Doi, M. \& Edwards, S. F. The Theory of Polymer Dynamics (Oxford University Press, Oxford, 1988).

5 McLeish, T. C. B. Tube theory of entangled polymer dynamics. Adv. Phys. 51, 1379-1527 (2002).

6 Ball, R. C., Doi, M., Edwards, S. F. \& Warner, M. Elasticity of entangled network. Polymer 22, 1010-1018 (1981).

7 Harada, A., Li, J. \& Kamachi, M. The molecular necklace: polyrotaxane containing many threaded alpha-cyclodextrins. Nature 356, 325-327 (1992).

8 Okumura, Y. \& Ito, K. The polyrotaxane gel: a topological gel by figure-of-eight crosslinks. Adv. Mater. 13, 485-487 (2001).

9 de Gennes, P. G. Sliding gels. Physica A 271, 231-237 (1999).

10 Kataoka, T. Mechanical properties of topological gel, MSc thesis, The University of Tokyo, Tokyo, 2003.

11 Mayumi, K., Endo, H., Osaka, N., Yokoyama, H., Nagao, M., Shibayama, M. \& Ito, K. Mechanically interlocked structure of polyrotaxane investigated by contrast variation small-angle neutron scattering. Macromolecules 42, 6327-6329 (2009).

12 Ito, K. Novel cross-linking concept of polymer network: synthesis, structure, and properties of slide-ring gels with freely movable junctions. Polym. J. 39, 488-500 (2007).

13 Bando, A., Kato, K., Sakai, Y., Yokoyama, H. \& Ito, K. Volume phase transition of slidering gels (to be submitted).

14 Shinohara, Y., Kayashima, K., Okumura, Y., Zhao, C., Ito, K. \& Amemiya, Y. Small-angle $\mathrm{X}$-ray scattering study of the pulley effect of slide-ring gels. Macromolecules 39, 7386-7391 (2006)

15 Mayumi, K., Nagao, M., Endo, H., Osaka, N., Shibayama, M. \& Ito, K. Dynamics of polyrotaxane investigated by neutron spin echo, Physica B. 404, 2600-2602 (2009). 